\title{
Philosophiques
}

\section{L'expression et les frontières de la réalité}

\section{Gilles Pronovost}

Volume 13, numéro 1, printemps 1986

URI : https://id.erudit.org/iderudit/203300ar

DOI : https://doi.org/10.7202/203300ar

Aller au sommaire du numéro

Éditeur(s)

Société de philosophie du Québec

ISSN

0316-2923 (imprimé)

1492-1391 (numérique)

Découvrir la revue

Citer cet article

Pronovost, G. (1986). L'expression et les frontières de la réalité. Philosophiques, 13(1), 3-20. https://doi.org/10.7202/203300ar

\section{Résumé de l'article}

De par sa nature essentiellement symbolique, l'expression vise à faire ressortir des éléments partiels ou globaux propres à une expérience originale de la réalité, par la médiation de formes diverses, dont les principales sont le langage, l'art et la science. Quant à cette dernière, l'auteur prend l'exemple de la sociologie, pour conclure que celle-ci constitue comme la limite épistémologique de la structuration des phénomènes sociaux, expression, dans un langage dit scientifique, des constantes de la vie sociale. d'utilisation que vous pouvez consulter en ligne.

https://apropos.erudit.org/fr/usagers/politique-dutilisation/ 


\title{
ARTICLES
}

\section{L'EXPRESSION ET LES FRONTIÈRES DE LA RÉALITÉ *}

\author{
par Gilles Pronovost
}

\begin{abstract}
RÉSUMÉ. De par sa nature essentiellement symbolique, l'expression vise à faire ressortir des éléments partiels ou globaux propres à une expérience originale de la réalité, par la médiation de formes diverses, dont les principales sont le langage, l'art et la science. Quant à cette dernière, l'auteur prend l'exemple de la sociologie, pour conclure que celle-ci constitue comme la limite épistémologique de la structuration des phénomènes sociaux, expression, dans un langage dit scientifique, des constantes de la vie sociale.
\end{abstract}

\begin{abstract}
As being essentially symbolic, the nature of expression consists in bringing out partial or global dimensions related to a peculiar experience of reality, through many forms, mainly langage, science, and the arts. As far as science is concerned, the author takes the example of sociology, and concludes that this discipline may be seen as the epistemological limit of the structure of the social phenomena, an expression, through a so-called scientific language, of the recurrent aspects of the social life itself.
\end{abstract}

\section{INTRODUCTION}

Nous utilisons tant de mots, et tant de voies, pour parler de tout et de rien, et nous avons parfois tant de difficultés, soit à nous comprendre, soit à exprimer clairement notre pensée, qu'on peut se demander pourquoi il en est ainsi, et surtout, ce que veut dire : parler. La question de savoir ce que nous voulons dire, quand nous nous exprimons, n'est pas vaine... Il y a tant de choses dans

* Ce texte est une version remaniée d'une communication présentée au colloque sur l'imaginaire, regroupement des professeurs en études québécoises, Université du Québec à Trois-Rivières, décembre 1984. 
le moindre geste, dans l'infime mouvement des yeux ou du corps. Il n'est pas jusqu'à notre silence qui ne parle tout haut.

Force est de reconnaître que parler fait problème. Ne rêvons-nous pas secrètement du jour où, du bout des doigts, d'un silence, d'un regard, la totalité d'une pensée pourrait être entièrement exprimée et comprise? Ne portons-nous pas en nous des traces d'indicible, comme un battement, comme un souffle qui meurt, et dont nous ne savons rien sinon qu'il est là, à attendre son heure, dans l'étincelle d'une pensée? Ainsi, non seulement nous souffrons parfois de mal parler, d'une sorte d'impuissance latente à nous exprimer correctement, voire à reconnaître la signification de notre parole, mais en plus nous sommes porteurs de choses inconnues.

Certes l'homme a inventé, et parfois avec beaucoup d'ingéniosité, différents moyens soit pour exsuder ses pensées, soit pour en resserrer le déploiement. Notre distance d'avec l'animal se traduit notamment par le langage, dont on a dit qu'il est cette institution spécifique à l'espèce humaine: l'homme, dès qu'il est connu, est un être expressif. Nous avons construit les différentes disciplines scientifiques, lesquelles traduisent une emprise incontestable sur la réalité. Nous utilisons divers procédés artistiques pour cerner ce monde qui nous entoure, ce monde dans lequel nous sommes vivants. Nous avons même, depuis la nuit des temps, laissé à quelques grands hommes, ou à d'obscurs interprètes, le soin de dire en notre nom, ce que nous sommes; nous avons produit une pléiade de savants, d'artistes, de poètes et de philosophes, qui nous ont informés un peu mieux sur la signification de notre réalité, ou encore sur la nature de notre destin.

Mais nous savons bien que tout cela ne suffit pas et ne suffira jamais. Nous sommes presque condamnés à parler toujours et toujours. Épisodiquement se lève un homme qui a tant et tant de choses nouvelles à nous dire. Et les librairies sont pleines de paroles qui n'attendent que notre regard...

On peut ainsi se demander: qu'atteignons-nous vraiment, quand nous tentons, par divers moyens ou procédés d'exprimer ou de comprendre la réalité extérieure? Quelle est la portée véritable du langage scientifique ou artistique? Jusqu'où va le sensé, où commence l'insensé ? Et y a-t-il des différences vraiment 
fondamentales entre les procédés dont use la science et ceux en usage dans le langage quotidien ou la pratique artistique?

Je n'ai pas, bien entendu la prétention de répondre à toutes ces questions. Elles forment néanmoins la trame fondamentale du questionnement auquel je veux m'attarder, sur la base de l'expérience et de la réflexion que $j$ 'ai sur une pratique scientifique particulière, soit celle du sociologue. Ce qui me semble en jeu, ce sont les possibilités mêmes, tout autant que les limites, de l'expression de la réalité extérieure.

\section{LA PRODUCTION ET L'EXPRESSION DE LA RÉALITÉ}

«Maintenant que notre coquille est détruite, qu'on est à un pas d'être partis des lieux et des objets où les jeux de l'habitude avaient tissé des toiles où faire courir des idées et des sentiments, maintenant qu'il ne reste plus rien de ça, on peut le dire sans se tromper: il n'y avait rien, il n'y a RIEN tout court. (...) Et là on voit, on sait, avec force, comme tout nus dans la neige, que ce qu'on est vraiment c'est un vide, que ce vide garde tout le temps sa force de vide, sa faim douloureuse, que ça dévore tout à mesure, nous avec. (...) On a trouvé qu'on est un vide qui se refait, que c'est ça notre sens et on est contents» (Réjean Ducharme) ${ }^{1}$

Dans le domaine des symboles et des significations, il existe un versant de la réalité, celui par lequel la connaissance ou la reconnaissance est donnée, les attributs et les qualités répartis entre les objets, les catégories de compréhension et d'interprétation réappropriées, ré-actualisées, selon les situations et les contextes. Bref on peut parler en ce cas du mouvement qui va des significations à l'acteur qui les reçoit ou les utilise. Mythes, représentations sociales, stéréotypes, catégories culturelles dominantes en sont des exemples.

Mais il existe aussi un autre mouvement; celui qui va de l'acteur aux significations qu'il produit, tente d'exsuder; tel est ce qui sera appelé: «expression». C'est le processus même de production des significations dont il s'agit cette fois. L'expression réfère au travail de production symbolique, aux divers procédés de construction de significations; elle vise à manifester expressément des significations, elle présente, extériorise des signes.

1. Ducharme, Réjean : L'biver de force. Paris : Gallimard, 1973 p. 180-181 ; les italiques sont de l'auteur. 
Il n'est pas suffisant d'exprimer quoi que ce soit; il faut aussi être entendu, produire du sens qui puisse être reçu. Se parler à soi-même suppose déjà un interlocuteur. C'est pourquoi on peut dire que l'expression vise fondamentalement la transformation d'une expérience originale en un système social de significations, son insertion dans une sorte de communauté des intelligibilités. En d'autres termes, la production des significations ne se déroule pas à vide, pourrait-on dire, mais dans le contexte d'un entendement minimal commun, d'un univers partagé de compréhension de ce qui est produit.

1. Le travail de l'expression puise ses matériaux premiers dans un univers de signifiants, déjà donnés; il est tributaire des intelligibilités déjà présentes dans la vie coutumière. L'expression exsude des éléments qui, à priori, sont déjà significatifs. Le poème n'existerait pas sans le langage, ni le roman bien sûr; mais que serait la danse sans référence aux gestes quotidiens, que serait donc la peinture sans la toile de fond des significations sociales courantes? Que serait la musique sans interprétation, et de l'exécutant, et du mélomane?

C'est en vertu de sa nature symbolique que l'expression, dans son travail ne peut d'abord faire ressortir que des éléments déjà signifiants. Rien n'est exprimé qui a priori ne soit significatif, un peu comme le signifiant et le signifié ne sont que les faces opposées d'un même signe. Prenons l'exemple de la musique; il y a tout d'abord la connotation même: place de la musique dans la vie sociale, différences de statut selon les milieux, styles, conceptions, etc.; il y a aussi les règles musicales, formes conventionnelles de l'écriture des sons; il y a les modalités d'exécution, le spectacle; et il y a l'interprétation des sons entendus («richesse», «beauté», "expression des émotions», "vivacité», etc.). L'expression musicale s'enracine dans des conventions sociales; on peut entrevoir l'étendue des définitions, l'ample tissu des significations, dont toute œuvre musicale est tributaire.

En généralisant ces propos, il appert que tout procès de production, sous quelque forme que ce soit, est d'abord inscrit dans un univers significatif, dans lequel d'ailleurs il puise ses matériaux essentiels. L'expression manifeste, extériorise, identifie des éléments qui ont déjà leur propre signification sociale; 
l'expression ne peut donc relever que d'une sorte de sélection particulière de certains signifiants puisés dans l'univers infini des signifiants de la vie quotidienne. Le discours poétique, par exemple, possède un «style» d'expression fondée sur des chaînes de mots structurées de manière souvent peu orthodoxe par rapport au discours commun, ou encore fondée sur des essais de fabrication de significations nouvelles par des utilisations de la connotation affective ou autre des mots quotidiens; il puise dans l'univers du langage ses matériaux premiers.

Toute activité de production symbolique est ainsi tributaire d'un univers de significations préalables, soit par les aspects de connotation auxquels elle participe, soit dans son utilisation de matériaux qui ne peuvent être que déjà signifiants (mots, gestes, formes, couleurs, etc.).

C'est pourquoi tout a déjà reçu quelque sens social, tout a déjà reçu un quelconque coefficient d'intelligibilité ; même ce qui est pensé comme «non-sens», «impensable» ou «inintelligible» a au moins cette signification particulière. L'activité symbolique de l'espèce humaine est totale, globale, rien ne lui échappe. La signification sociale est déjà en place au moment de la première activité expressive. Pour la raison que l'expression est liée comme son envers à la signification sociale. L'univers entier ne peut donc qu'être, dans sa totalité, significatif. En d'autres termes, il n'est pas possible, au moins pour l'espèce humaine, d'exister sans un univers de significations préalables; on pourrait dire que l'espèce humaine et l'univers significatif sont comme les deux pôles complémentaires d'une même réalité, par analogie avec le signifiant et le signifié, pôles indissociables d'un même signe...

L'expression est ce long exercice d'appropriation, de mise à jour, de reformulation des significations sociales dont notre univers est tissé.

L'expression vise ainsi à puiser dans des matériaux déjà signifiants pour exsuder tout le sens dont l'homme social est capable, comme pour ajouter de nouvelles dimensions significatives qui auraient été oubliées, comme pour parcourir. la densité symbolique de l'univers social en la balisant de nouveaux repères significatifs. 
Dans ce premier moment de l'étude de l'expression, celle-ci ne serait donc pas possible sans cette signification préalable de l'ensemble de la réalité sociale; en ce sens la «création", au sens strict, est impossible, puisque la seule activité d'invention ne peut reposer que sur l'utilisation nouvelle de possibilités déjà données a priori, que sur des combinaisons de signes déjà là, que sur une grille de référence déjà construite, que sur une signification première dont elle est marquée.

Mais l'on sait que l'univers social des signifiants, d'où l'expression tire ses matériaux, est relatif et changeant. Nous savons bien que, connaissance sociale, attributs, catégories de pensées, arguments, mythologies, bref que tout le contenu significatif propre à une époque donnée, a passé ou passera. La totalité des significations que véhicule une société est appelée, tôt ou tard, à se transformer. Même la science n'échappe pas, dans sa constitution, son contenu et son développement, à cette historicité, car nous savons aussi que les modèles, les théories et les concepts basculent un jour dans le passé, dans l'inexactitude ou l'erreur. En ce sens, si le monde social est le seul monde connaissable et exprimé, il est arbitraire... Il n'existe pas bien entendu de paramètres nous permettant de définir que telle culture, telle représentation est plus «objective» qu'une autre, à moins de faire appel à des garants méta-sociaux. Il n'existe pas de poésie définitive, non plus que de musique infinie. Il n'existe pas non plus d'univers social achevé. La connaissance que nous avons du monde social est conjonctuelle, partielle et passagère, sans que l'on puisse entrevoir comment échapper, si besoin en est, à cette situation.

Prophètes et prophéties, mythes et idéologies, dieux et déesses, viennent souvent s'engouffrer dans cette douleur de l'incertitude et de l'arbitraire.

Et même le sociologue, dans son expression de la réalité sociale, n'élabore certes pas une sorte d'autre social, qui serait une image plus fidèle du monde. L'historicité et l'arbitraire de l'univers social ont pour corrolaire la fragilité voire la mouvance des sciences sociales elles-mêmes, c'est-à-dire : les déplacements de point de vue, de perspectives, les modifications des concepts et des théories, la vétusté ou le parti-pris des interprétations. 
C'est pourquoi la seule issue possible nous semble la suivante: parcourir sans fin, l'univers social, le monde des significations, grâce aux diverses modalités d'expression symbolique. Dire et redire du sens ; qui plus est, l'objectif avoué de maintes expressions symboliques est de dépasser l'horizon culturel d'une civilisation, aller au-delà des limites du sens, (roman, poésie, science-fiction, etc.). Mais cette intention fait partie intégrante de notre univers; «il n'y a plus rien» est aussi contenu dans un espace de signification. Et à l'infini.

2. Mais cela ne doit pas s'interpréter comme l'impossibilité absolue de «création». De toute façon, nous faisons régulièrement l'expérience de la capacité créatrice de l'espèce humaine, non pas cette fois par le surgissement, du fond de notre culture, de contenus latents ou inconscients qui savent nous émouvoir par leur justesse, mais par l'identification relative de sens nouveaux soumis à l'attention des hommes. Il y a des activités de production de symboles qui conduisent à des transformations radicales de notre compréhension, voire de notre connaissance; c'est-à-dire qu'il y a des procès de constitution de sens nouveaux, signe d'une véritable capacité créatrice de l'espèce humaine.

C'est dans la relativité de l'univers social, dans son historicité, que le sens nouveau prend forme. Les cultures ne sont jamais à ce point refermées sur elles-mêmes qu'elles puissent dénier le changement. La production culturelle est cette ouverture vers les possibilités d'innovation radicale, contrepartie négative de la stabilité d'une société.

Les contrôles sociaux ne suffiront jamais à contenir dans les limites du déjà dit le sens dont nous sommes tissés, et ce, par-delà l'impératif absolu, pour toute société de parvenir à un certain équilibre. Chaque mot, chaque parole, peut être source de tensions. Et même si nous nous taisions tous, l'expression surgirait encore, des regards, des gestes, des mouvements; il y a une contradiction fondamentale entre les impératifs propres au maintien, à la consolidation, à la permanence relative des structures sociales, et l'irrésistible quête d'expression.

Ce qui est «nouveau» résulte du fait que les sociétés sont de véritables passoires; elles ne peuvent contenir les changements qui se profilent de toutes parts. Tout ce qui n'a pas encore été dit 
manifeste la précarité, le caractère transitoire des sociétés constituées.

L'identification de la nouveauté de significations produites est sujette à son tour, à des procédés de définition sociale. Non seulement y a-t-il enjeux autour du «droit » à la créativité, mais il y a aussi des règles de normalité. L'expression culturelle de sens nouveaux se voit dès lors doublée d'une sorte de coefficient d'interprétation, puisqu'elle suppose un minimum de conventions sociales quant à sa production et à sa compréhension. Il n'est qu'à écouter les critiques d'art pour s'en convaincre.

En plus, donc, de constituer une opération combinatoire de signifiants déjà donnés, à partir de laquelle on ne peut conclure qu'à l'arbitraire de toute parole sur le monde, et à l'arbitraire de toute culture, il existe manifestement certaines activités expressives qui donnent lieu à des productions inédites de sens nouveau. Mais ici il faut départager la «nouveauté» identifiée en fonction des modèles culturels et des conventions sociales, de celle irréductible à toute identification passée, c'est-à-dire de cette nouveauté, unique, individualisée, issue de la pratique empirique. Le problème majeur qui se pose est celui des moyens dont on peut disposer pour départager l'activité combinatoire, les modèles culturels d'expression, et les significations sociales uniques issues d'expériences empiriques uniques. Tout ce que nous pouvons dire, c'est que nous savons que la praxis humaine est le lieu de la fabrication d'expériences nouvelles et de traductions symboliques inédites; et qu'en plus de cette totalité de significations sociales, dont nous sommes porteurs, dont nous tentons inlassablement de nous approprier, vient «s'ajouter» en quelque sorte un discours nouveau, ouvert à ces signes inconnus qui surgissent de l'expérience humaine, mais que nous avons tant de mal à identifier.

3. Ceci étant dit, nous pouvons maintenant interpréter ainsi la double fonction individuante de l'expression.

Il y a celle qui vise d'une part à l'individuation d'un contenu significatif; par divers procédés, les acteurs sociaux fabriquent des chaînes de significations particulières, mais, ce qui est capital, à partir d'un univers de signifiants déjà donnés, à partir de matériaux divers utilisés à titre de signifiants. L'expression tend à isoler des pièces significatives, des morceaux de sens; l'expression 
identifie dans l'univers du sens des points d'attache symbolique, des points de repère, elle porte à l'attention des acteurs des ensembles de significations.

Mais nul ne peut se satisfaire de telles significations ponctuelles. L'expression est aussi une quête de totạlité de significations, la recherche du sens global à donner aux choses et aux objets. L'expression est la voie qu'emprunte le fait social pour se donner une figuration d'ensemble de lui-même. Comme l'écrit Gadamer, en parlant de la science :

«... il y a une anticipation du tout, qui précède déjà et englobe toute les schématisations particulières de la science. C'est le langage dans lequel nous grandissons, nous parlons et nous nous entendons les uns avec les autres, en agissant sur nous-mêmes et sur notre monde» (1982, p. 21).

L'art également, est fait de telles recherches d'appréhensions globales, de cette totalité à laquelle renvoient et le langage et toute expérience individuelle.

C'est une des caractéristiques profondes de l'expression que de se porter à la fois vers l'identification de toutes les significations particulières dont l'univers peut être tissé, et de sa signification dernière. Peine perdue, d'ailleurs, puisque l'espèce humaine, depuis qu'elle est connue, n'a pas cessé son travail d'expression, et qu'on ne voit pas le jour où elle cessera. Il y a dans l'expression, une sorte de douleur à ne pouvoir, une fois pour toute, contenir la signification de l'univers social; et malgré cela, sans qu'on n'entrevoie jamais la fin d'une telle tentative, l'espèce humaine continue de s'exprimer. Et chaque parole s'ajoute en quelque sorte, à la réalité sociale, vient en accroître la complexité et la densité.

4. Nous avons là, croyons-nous, les principaux éléments nécessaires à la poursuite de notre cheminement. Une sociologie de l'expression ne peut que faire d'abord appel à des constantes et des modèles culturels, tout comme elle peut indiquer, quand cela lui est possible, quelles sont les voies par où, pour l'instant la réduction analytique est impossible. C'est dans ce double mouvement des contrôles sociaux de l'expression et de l'ouverture à la véritable innovation, que se situe notre démarche: formes et modalités sociales de l'expression, limites actuelles du tracé 
analytique : structures de la création culturelle, débordement vers l'historicité.

\section{FORMES DE MÉDIATION DE L'EXPRESSION}

L'expression symbolique emprunte diverses modalités, s'actualise, se manifeste, de diverses manières. Il n'y a pas d'expression sans une quelconque forme expressive. L'expression est toujours médiatisée.

1. - La forme la plus simple, la plus commune, et la plus essentielle, est le langage. Ce n'est pas un hasard d'avoir à la rappeler, puisqu'on sait que le langage constitue l'invention la plus originale de l'espèce humaine, est la manifestation la plus radicale de ses rapports avec l'environnement, par ce processus de dédoublement symbolique dont on a déjà parlé. Le langage est la forme première de la socialité. En conséquence, toute recherche sur l'expression suppose un minimum d'attention au langage. Comme ce n'est pas l'objet de cet essai nous ne ferons que souligner ceci :

«... le langage est la forme des formes, il est ce qui fait que n'importe quelle forme est médiatisée en elle-même, inclut sa propre réflexion dans le mouvement par lequel l'existence humaine tente ses propres possibilités. Le langage, en tant qu'il est constitutif de la société proprement humaine, est forme médiatrice entre l'expression et la signification» (ORTIGUES, 1962, p. 25).

À l'intérieur du langage, le discours, en particulier, est une forme d'expression régie par un mode de communication proprement linguistique (ibid., p. 24).

2. La science est aussi une forme d'expression originale, et relativement circonscrite; elle s'appuie sur une tradition de recherche, des procédés spéciaux d'entrée en contact avec le réel, des techniques d'expérimentation, et un vocabulaire spécialisé. Sa particularité la plus remarquable, et, fondamentalement, celle qui la définit, est qu'elle tente de lier de la façon la plus adéquate possible, les signifiants et les signifiés; elle vise la construction d'une sémantique qui soit formellement appropriée aux référents empiriques; en d'autres termes, le langage qui lui est propre veut dénoter de la façon la plus stricte possible le compte-rendu des données de l'expérience (voir GRANGER, 1965); elle y parvient 
à l'aide d'un univers sémantique rigoureusement construit et d'un contrôle des données de l'expérience (v.g. expérimentation en laboratoire). La science nous semble la seule forme d'expression qui puisse poser de façon adéquate le problème de la référence; mais c'est au prix de la double manipulation et des symboles et des données empiriques; hors de cela, les relations entre les éléments signifiants et l'expérience à laquelle ils peuvent référer demeurent toujours imparfaites, inadéquates, ambiguës même, et dans le cas de l'art elles sont parfois franchement inutiles.

On peut à bon droit se demander si ce que tente d'exprimer la science n'est que l'image de ses propres constructions sémantiques, voire le reflet de son langage particulier (langage souvent entremêlé de langages autres: langage quotidien, idéologies). Si un tel péril est constitutif de la science, la contrepartie nous semble son indiscutable emprise relative sur les phénomènes empiriques, puisque la science est capable d'applications.

3. La troisième grande forme d'expression que nous voulons identifier est l'art. Celui-ci est un procédé qui consiste à lier de quelque manière un élément quelconque, qui agit à titre dé signifiant, et son contenu idéel tout aussi quelconque.

«... l'art est lui-même, dans une certaine mesure, une technique sur le double plan des activités opératoires et figuratives. (...) on n'explique pas un langage en fonction des choses qu'il nomme ou des rapports d'idée qu'il exprime. Le but de l'art n'est pas de constituer un double maniable de l'univers; il est, à la fois, de l'explorer et de l'informer d'une manière nouvelle. La pensée plastique qui existe à côté des pensées scientifiques ou techniques appartient à la fois au domaine de l'action et de l'imagination. »(FRANCASTEL, 1970, p. 13).

Il faut faire remarquer ce qu'on pourrait appeler sa pédagogie particulière de production symbolique. En premier lieu, l'art est très près de l'imagination sensible; il s'attache à une densité de sentiments et d'émotions, qu'il tente d'extérioriser par des symboles naturalistes, matériels, par des appels à des éléments concrets, transposés sous forme concrète (v.g. gestes, formes matérielles) ou très abstraite (c.f. un poème de Paul Valéry); ou encore, il utilise des symboles stylisés, épurés, dans des formes concrètes d'expression (v.g. la musique qui, par des sons veut exprimer des sentiments). L'étendue de l'expression artistique va 
ainsi de l'abstraction la plus élevée à la concrétude et la sensibilité les plus poussées.

Ce qui introduit à une deuxième caractéristique : l'art multiplie à l'infini la gamme des éléments pouvant faire l'objet d'une appropriation symbolique, ou d'utilisation à des fins de signification: sons, couleurs, formes, matériaux (bois, verre, tissu, métal, etc.), signes linguistiques, gestes et mouvements du corps. Tout est susceptible de transposition artistique. L'art nous semble l'activité ultime de manifestations du symbolisme inhérent à la socialité humaine, la preuve infinie de l'importance de la production symbolique.

L'art contemporain, pour sa part, se caractérise en plus par ce qu'on a appelé la disparition de la référence (KLEIN, 1970). L'exemple typique est la peinture dite abstraite: renoncer à l'image, ne vouloir rien dire, à la limite, que son intention de faire « œuvre d'art». L'art moderne rejette toute référence hors de lui-même; l'œuvre est à elle-même sa propre fin, elle se pose comme le commencement et le terme de sa «signification». L'artiste moderne ne s'occupe plus du symbole (v.g. la couleur ou la forme) et de ce qu'il pourrait éventuellement signifier; et effectivement, les toiles «abstraites» ne veulent rien dire, si ce n'est leur intention d'avoir été produites.

En d'autres termes, l'artiste délaisse la référence, pour s'attacher à l'opération de création; la production artistique porte sur un aménagement des signifiants - qui sont souvent constitués de matériaux divers - auxquels sont indissolublement liés des signifiés, mais la quête d'une éventuelle signification ou la recherche de la référence empirique, constituent des tâches sans fin; l'artiste contemporain a compris la part d'arbitraire des significations sociales, et les rejetant, s'attarde plutôt à ce qu'on pourrait appeler la grammaire du sens, se replie sur le geste même de création de symboles, indépendemment de leur portée significative; si l'art est une manière d'exprimer n'importe quoi avec.n'importe quel matériau retenu au seul titre de signifiant, l'artiste a compris que c'est l'exprimer qui est important, non le «n'importe quoi», ni même le signifiant. Telle est la définition la plus fondamentale de la création artistique: l'opération même d'expression, quels qu'en soient les résultats. 
Ce faisant, on peut se demander si l'art n'est pas à inscrire dans une ambiguïté profonde d'où il ne peut échapper: soit se définir comme pure activité combinatoire de signifiants, et donc renoncer au sens, - telle toile n'est pas à comprendre, mais à voir - soit vouloir dire du sens, mais sans pouvoir le reconnaître vraiment : combien de poèmes n'échappent-ils pas à leur auteur?

\section{L'EXEMPLE DE LA SOCIOLOGIE}

On peut maintenant se demander ce qu'exprime réellement la sociologie, quand elle traite des phénomènes sociaux. Je prends cet exemple parce qu'il s'agit de ma discipline de formation, et aussi parce qu'il s'agit d'un «cas» fort instructif quant aux possibilités qu'ont les sciences sociales de prétendre à une quelconque emprise sur le réel.

Si l'on revient au point de départ, les questions que l'on peut se poser, par rapport au travail du sociologue, peuvent se résumer ainsi: dans quelle mesure les concepts sociologiques constituent des productions de significations « nouvelles», ou plutôt des réappropriations de significations déjà construites; ou en d'autres termes: dans quelle mesure les concepts sociologiques sont des inédits ou des calques des modèles culturels dominants. Une autre question que l'on peut se poser réfère encore au rôle que joue la communauté scientifique des sociologues dans la reconnaissance, l'acceptation ou le refus des productions sociologiques; c'està-dire, aux procédés d'attribution de la légitimité accordée ou non à tel ou tel travail sociologique, ce que nous avons appelé: la reconnaissance sociale de la nouveauté.

Il n'a pas échappé non plus à maints observateurs l'existence de tensions constantes, en sociologie, entre les analyses particulières et les tentatives récurrentes de généralisations, d'interprétations globales, c'est-à-dire ce que nous avons appelé: la double fonction individuante de l'expression.

On reconnaîtra que de telles questions nécessitent un examen détaillé, soigné, qui dépasse le cadre de ce texte. C’est pourquoi nous ne retiendrons qu'un aspect, aussi crucial, celui de la pertinence des concepts sociologiques par rapport aux phénomènes sociaux qu'ils prétendent décrire, sinon expliquer. Formulée 
dans des termes analogues à ceux qui ont été utilisés pour l'étude de l'expression, la question peut se formuler ainsi : quels sont les rapports qu'entretiennent les concepts sociologiques avec la réalité sociale déjà structurée, et dans quelle mesure l'historicité de toute situation sociale ne rend pas précaire, sinon inutile, les tentatives de construction des concepts sociologiques, appelés tôt ou tard à devenir caduques?

Nous sommes ici, comme dans toutes les sciences humaines et sociales en présence d'une diversité de niveaux d'analyse, d'interprétations et d'hypothèses; de plus la sociologie de la connaissance nous apprend que le sociologue-observateur fait aussi partie de l'observation, et que ses concepts sont en partie relatifs à sa culture... Ce qui signifie, tel que je l'ai évoqué au tout début que si les concepts sociologiques sont foncièrement de nature historique et culturelle, la structuration de notre compréhension de l'univers social à laquelle ils nous invitent, est arbitraire. Car non seulement les structures sociales changent, mais aussi les catégories de l'analyse sociologique. Dans ce dernier cas, les déplacements de la production sociologique sont en grande partie tributaires des transformations des sociétés contemporaines elles-mêmes. Et il n'y a pas de "point d'appui» permettant d'affirmer la pertinence ultime, la justesse absolue, de tel ou tel concept sociologique. La sociologie est une discipline en tension constante entre la fidélité aux phénomènes sociaux, par des observations les plus pertinentes possibles, et la critique des structures en place; elle nous fait étudier soigneusement des phénomènes que nous savons passagers, sinon à radicalement transformer.

On peut ainsi se demander ce qui est réellement désigné par ce terme de «social»: s'agit-il d'une dimension du réel qui ne peut être approchée que de façon détournée? S'agit-il d'un objet fugitif et changeant que l'on tente de circonscrire de toutes parts et de multiples manières ?'Y aurait-il, comme caché, une sorte de réalité sociale «ultime», "vraie», que l'expérience sociologique tente d'aborder par concepts interposés? Ou encore: Ce social constituerait-il une sorte d'objet informe, insaisissable, une sorte de matière brute que le sociologue tente de domestiquer par une connaissance fragmentée et mouvante? 
Cette question de la nature ultime du social, et cette autre des attributs objectifs et fondamentaux de la réalité sociale, n'ont pas été souvent explicitement discutées. Je les ai soulevées, ne fût-ce que rapidement, pour souligner qu'elles font surgir ce débat stratégique, de la distinction entre les concepts et la «réalité». Or, fréquemment, le débat est trop rapidement esquivé en sociologie par cette distinction cartésienne entre une réalité sociale, mouvante, inatteignable, inconnaissable, et des concepts sociologiques qui en constituent une sorte d'image ou de dédoublement, une sorte de "social de second degré» qualitativement différent de quelque chose que l'on ne connaîtrait jamais dans toute son étendue.

Si le réflexe est courant, et, dans une certaine mesure, légitime, le danger qu'il soulève est immense, car il suppose implicitement la représentation d'une sorte de «dualité fondamentale», en vertu de laquelle il y aurait, d'une part, la réalité empirique connaissable, certes, mais fuyante et au fond à jamais complètement inaccessible, et, d'autre part, des concepts qui, plus que de simples vues de l'esprit, constitueraient au mieux une image partielle, mais «contrôlée», de la réalité.

Je suis d'avis que nous devons refuser cette approche cartésienne. Il me semble qu'il n'y a pas, d'une part, le social « réel», empirique, riche et touffu, et d'autre part, un concept du social, sorte de "social théorique», et qui constituerait comme une image objective, stylisée, comme une sorte de modèle approximatif du premier et vers lequel la science tendrait en resserrant peu à peu son étreinte.

Je ne pense pas qu'il y ait des différences vraiment fondamentales entre le social que recherche le chercheur et les concepts qu'il vous livre à la suite de ses efforts d'analyse.

A l'appui de cette thèse, l'argument le plus important me semble le suivant : les concepts explicatifs, les modèles prédictifs, les constantes, voire les déterminants que recherche le chercheur dans son étude du social, seraient dénués de sens si, effectivement, les sujets individuels ou les institutions sociales n'agissaient pas ainsi. Les explications et les interprétations de la science tentent en effet d'en arriver à prédire ce qui devrait se passer dans telle ou telle situation, compte tenu de divers facteurs; la sociologie, à son 
tour, vise précisément à trouver les modalités et les lieux d'observation de telles prédictions. Or une telle recherche de facteurs explicatifs et prédictifs s'appuie tout simplement sur le fait qu'il $y$ a des constantes dans la vie humaine: les corrélations statistiques, la recherche des structures de l'action sociale, les modèles psychologiques ou économiques, autant d'exemples qui se fondent sur l'existence de constantes et de régularités. La sociologie est la reformulation, selon les règles classiques de la méthode scientifique (vocabulaire univoque et clair, procédés de vérification, etc.) de la démarche des acteurs sociaux eux-mêmes.

Selon des termes déjà évoqués, l'expression de nature sociologique s'alimente aux constantes mêmes de la réalité sociale. Elle est un processus d'identification, de mise à jour, des structures dont la réalité sociale est tissée. Le discours sociologiques procède d'une sorte de sélection plus ou moins arbitraire des signifiants puisés dans l'univers à la fois stable et mouvant de la vie quotidienne, il puise dans la réalité sociale ses matériaux d'expression.

L'objectivité du social ne peut se fonder sur une dualité réalité-concept, dans laquelle l'objectivité serait du côté du concept, et la réalité apparaîtrait comme quelque chose d'informe... Je dirai plutôt que l'objet fondamental de la sociologie est non pas le social lui-même, mais ce que j'appelerais : son objectivité radicale.

Si donc quelqu'un se demande: mais qu'est-ce donc que cet objet dont nous parle le sociologue? Mais qu'est-ce donc que cette chose dont il est question dans la sociologie? La réplique devra faire appel à l'objectivité essentielle de toute situation sociale, tout autant qu'à son caractère passager et arbitraire.

De tout cela il s'ensuit une conséquence importante. La sociologie fait partie intégrante du social lui-même, non seulement en vertu de l'axiome bien connu selon lequel le chercheur des sciences humaines fait lui-même partie de son champ d'observation, mais aussi parce qu'elle constitue comme la limite de l'organisation objective du social. La sociologie est l'expression dans un langage particulier qu'on appelle scientifique, des constantes de la vie quotidienne, des règles d'action sociale, des modalités de fonctionnement des institutions. La sociologie est la 
mise en forme objective de l'objectivité du social. Ses méthodologies, ses techniques, ses concepts et ses théories, constituent comme la limite épistémologique de sa structuration.

Si une dualité commode avait à être évoquée, ce ne serait certes pas entre le social-phénomène et le social-concept, puisque le second est un élément constitutif du premier. On songerait plutôt à la distinction science-objectivité, en vertu de laquelle serait dit: "science», tout procédé s'appuyant sur les usages courants de méthodologie, de définition rigoureuse des concepts, d'expérimentation contrôlée, etc.; et serait dit «objectif» tout trait constant de la vie sociale; en ce cas, le scientifique serait du côté de la science, et l'objectif du côté de ce que, faute de mieux, on peut désigner par «la réalité». Dans la mesure où la réalité du social ne comporte pas que des aspects objectifs, il appert qu'il faut aussi faire appel à autre chose que la science pour en parler.

\section{CONCLUSION}

A partir d'une interrogation sur les possibilités mêmes qu'a l'espèce humaine de pouvoir exprimer de manière significative la réalité extérieure, il est apparu que la production des significations était soumise à des conditions sociales, historiques et culturelles dont on ne peut qu'entrevoir les difficultés, voire l'arbitraire. Mais puisque nous tentons malgré tout, indéfiniment, d'exprimer et de comprendre le monde qui nous entoure, l'expression oscille entre l'individuation des significations et la recherche de totalités significatives.

D'autre part, il est manifeste que nous utilisons des modalités, des registres fort divers, mais non contradictoires, d'expression. On peut sommairement et classiquement; distinguer: le langage, l'art, la science.

Pour ce qui est de cette dernière, nous avons rapidement pris l'exemple de la sociologie pour illustrer qu'à l'instar du langage, de l'art, cette discipline de recherche oscillait à son tour entre des formes singulières et globales d'expression de cette réalité particulière, qu'est la réalité sociale. Et, à l'instar de l'art, notamment, tout comme il n'y a pas de phénomènes de productions artistiques qui ne soient, fondamentalement des phénomènes d'appropriation 
de l'une ou l'autre dimension de notre univers, de même il n'y a pas de concepts sociologiques qui, de manière même obscure, détournée, voire parfois franchement malhabile, n'exprime quelque facette de la vie sociale.

Comme l'écrit Wittgenstein, «il est évident que si différent que puisse être du monde réel un monde imaginé — il doit encore avoir quelque chose de commun - une forme avec le monde réel» (1961, par. 2.022). Cette « forme», qui consiste en « objets», on peut la traduire encore par : constantes, objectivités, structures. Parvenir aux formes, c'est atteindre les limites de ce que la science peut dire, et en même temps, les limites des structures arbitraires de l'instant.

Département des Sciences du loisir

Université du Québec à Trois-Rivières

\section{BIBLIOGRAPHIE}

FranCASTel, Pierre. Etudes de sociologie de l'art. Paris: Denoël-Gonthier, 1970, $252 \mathrm{p}$.

GADAmer, H.G. L'art de comprendre. Paris, Aubier, 1982, 295 p.

Granger, Gilles-Gaston. Essai d'une philosophie du style. Paris: Armand Colin, 1968, $310 \mathrm{p}$.

KLEIN, Robert. La forme et l'intelligible. Paris, Gallimard, 1970, 688 p.

LANE, Gilles. L'avenir d'une prédiction. Montréal, Presses Universités du Québec, 1971, 159 p.

LÉvi-Strauss, Claude. La pensée sauvage. Paris, Plon, 1972, 395 p.

ORTIGUES, Edmond. Le discours et le symbole. Paris, Aubier, 1962, 228 p.

Rivière, Claude. L'objet social. Paris, Marcel Rivière, 1969, 380 p.

TINLAND, Frank. La différence anthropologique. Paris, Ed. Aubier-Montaigne, $1977,653 \mathrm{p}$.

WitTgenstein, Ludwig. Tractatus logico-philophicus. (Idées), Paris, Gallimard, 1961. 